\title{
Patientenverfügung und Demenz - Fälle, die sich schwer entscheiden lassen?
}

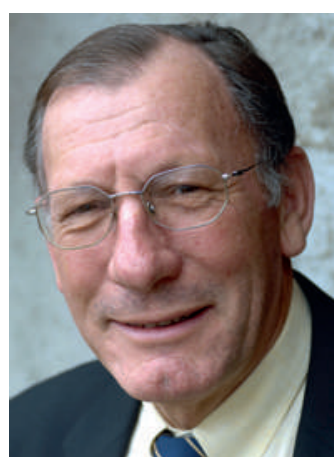

Jean Martin
Wie allgemein bekannt, sind Patientenverfügungen (PV) seit dem 1. Januar 2013 im Schweizerischen Zivilgesetzbuch (neue Bestimmungen zum Erwachsenenschutzrecht) verankert. Sie sind - wenn man so will ohne Bedeutung, so lange der Patient bei Verstand ist. Dann zählt, was er im jeweiligen Moment äussert. Verbindlich werden sie aber, wenn der Verstand getrübt ist.

Patientenverfügungen haben eine Debatte ausgelöst, die offensichtlich noch lange nicht beendet und mit folgender Fragestellung verknüpft ist: Wie soll man wissen, ob die Person in dem Moment (in dem sie nicht mehr bei Verstand ist), nicht anders entscheiden würde, als sie dies zuvor in der Verfügung ausgedrückt hat? Nach welchen Kriterien dürfen die Angehörigen und das Pflegeteam «urteilen», ob der Kranke seine Meinung und seine Geisteshaltung möglicherweise geändert hat?

Die Problematik liegt in der möglichen Differenz zwischen dem then-self (dem früheren Ich bzw. Selbst) und dem now-self. Gehen wir ein Risiko ein, wenn wir uns im Falle der Demenz auf das then-self verlassen, das die PV abgefasst hat? Die nationale Ethikkommission hat sich dieses Themas angenommen und 2011 einen Bericht veröffentlicht, der insbesondere auf die Demenz fokussiert. Dort heisst es: «Die Kontinuität der Person ist eine normative Grundvoraussetzung für die Gültigkeit einer Patientenverfügung. Die Kommission geht davon aus, dass diese Kontinuität auch im Falle der Demenz weiterhin gegeben ist.» [1] Neulich las ich ein Buch, in dem sich drei belgische Ethiker mit Fragen zum Lebensende befassten [2]. Chris Gastmans thematisiert den Respekt vor der Würde dementer Patienten, wenn diese Patientenverfügungen aufgesetzt haben, in denen sie nach Sterbehilfe verlangen. Anmerkung: In der Schweiz präzisiert das neue Recht, dass Patientenverfügungen keinen begleiteten Suizid und schon gar keine Sterbehilfe fordern können, wie dies z.B. in den Niederlanden möglich ist. Am Ende einer von der sog. Ethik der Fürsorge (Care ethics) geprägten Diskussion schliesst Gastmans mit der Aussage «Advance euthanasia directives do not meet the essential criteria of dignified care». [3] Im selben Werk nimmt den Hartogh [4] eine andere Position ein und betont, dass wir das Recht haben, nach von uns geäusserten Ansichten behandelt zu werden, selbst wenn sich diese als falsch erweisen sollten [4, 5].

Dabei fällt eine Bemerkung auf, die unmittelbar auf den vorstehenden Satz Gastmans folgt: «Nevertheless, further ethical analysis is needed, not the least because dementia is becoming more prevalent.» Lässt der
Autor hier - ohne ihn überinterpretieren zu wollen die Tür zu einer anderen Schlussfolgerung offen, die den vom Kranken geäusserten Willen respektieren würde? Aus quantitativer Sicht und unter dem Blickwinkel ihrer Prävalenz in der Bevölkerung wird Demenz das mit knappen Ressourcen ausgestattete sozialmedizinische System in nicht ferner Zukunft tatsächlich erschüttern. Dies in dem Sinne, dass der Nutzen einer Vorgehensweise im Verhältnis zu anderen Aktionen, die dieselben Ressourcen nutzen (immer mehr) berücksichtigt werden müsste.

Die Fondation Chrysalide, eine Einrichtung zur Palliativpflege in La Chaux-de-Fonds, brachte jüngst ein interessantes Heft [6] heraus, in dem verschiedene Dimensionen des Lebensendes betrachtet werden. Der Seelsorger G. Berney äussert seine Bedenken bezüglich der PV und meint: «Zweifellos haben sie ihre Berechtigung, und dennoch würde ich Abstriche machen. Idealerweise können wir jenen vertrauen, die wir lieben, denn mit ihnen konnten wir bereits vor dieser letzten Phase über unsere Bedürfnisse und unsere gegenseitigen Erwartungen sprechen.» Dies gilt sicherlich für den Idealfall, von dem wir hoffen, dass er häufig vorkommt. Was aber ist mit den anderen Fällen? Berney zitiert die Anthropologin Yvonne Preiswerk: «Was auch immer die Verstorbenen zu Lebzeiten gesagt haben, entscheiden die Lebenden.» Ich habe zwar weniger Erfahrung als diese Experten, dennoch halte ich die jüngst eingeführten Rechte des Patienten, die sich grob in der Formel zusammenfassen lassen «die anderen (med. Personal, Angehörige) schlagen vor, der Kranke entscheidet», für wohlbegründet.

Fazit: Es gibt ein Spannungsfeld zwischen jenen Ärzten, Pflegenden und Ethikern, die vorwiegend auf die Ethik der Fürsorge setzen, auf die Berufung bzw. das Mitgefühl, das ihrer Rolle inhärent ist, und jenen, die vor allem die Autonomie des Kranken betonen und daher nicht über seinen zum Ausdruck gebrachten Willen hinausgehen wollen. Im praktischen Alltag sind die Dinge nicht eindeutig schwarz oder weiss zu sehen. In Grenzfällen kommt für gewisse Fachleute eher die erste Erwägung zum Tragen, für andere die zweite. Es gibt Fälle, in denen nicht entschieden werden kann und doch entschieden werden muss. Die klinische und menschliche Erfahrung, der Respekt vor dem Kranken, praktisches Wissen (Phronesis/Prudentia) und der gesunde Menschenverstand ermöglichen das Handeln - oder auch Nicht-Handeln - zum Besten des Patienten.

Jean Martin, Mitglied der Redaktion 